\title{
Beyond Depression? A Review of the Optimal Foraging Theory Literature in Zooarchaeology and Archaeobotany
}

\author{
Emily Lena Jones ${ }^{1 *}$ and David A. Hurley ${ }^{2}$ \\ ${ }^{1}$ Department of Anthropology, University of New Mexico, Albuquerque, NM, USA. ${ }^{2}$ University Libraries, University of New \\ Mexico, Albuquerque, NM, USA. \\ *elj@unm.edu
}

\begin{abstract}
The use of optimal foraging theory in archaeology has been criticized for focusing heavily on "negative" humanenvironmental interactions, particularly anthropogenic resource depression, in which prey populations are reduced by foragers' own foraging activities. In addition, some researchers have suggested the focus on resource depression is more common in the zooarchaeological literature than in the archaeobotanical literature, indicating fundamental differences in the ways zooarchaeologists and archaeobotanists approach the archaeological record. In this paper, we assess these critiques through a review of the literature between 1997 and 2017 . We find that studies identifying resource depression occur at similar rates in the archaeobotanical and zooarchaeological literature. In addition, while earlier archaeological applications of optimal foraging theory did focus heavily on the identification of resource depression, the literature published between 2013 and 2017 shows a wider variety of approaches.
\end{abstract}

Received September 5, 2016

OPEN ӘACCESS

Accepted March 10, 2017

DOI 10.14237/ebl.8.1.2017.786

Keywords Resource depression, Optimal foraging theory, Prey choice, Zooarchaeology, Archaeobotany

Copyright (c) 2017 by the author(s); licensee Society of Ethnobiology. This is an open-access article distributed under the terms of the Creative Commons Attribution-NonCommercial 4.0 International Public License (https://creativecommons.org/licenses/by-nc/4.0), which permits non-commercial use, distribution, and reproduction in any medium, provided the original author and source are credited.

Supplementary Files available at ojs.ethnobiology.org/index.php/ebl/article/view/786

\section{Introduction}

The debate on the use of optimal foraging theory in archaeology-particularly in zooarchaeology and archaeobotany-has been heated in recent years, with critiques emerging in a variety of different contexts (e.g., Codding et al. 2010; Jones 2016a:9-22; Reitz et al. 2009; Smith 2015; Speth 2013; Zeder 2012, 2015a, b). While these critiques vary both in their natures and in the bases of their arguments, many of them concern, at least in part, the use of foraging theory to identify cases of prehistoric resource depression, or decreases in foraging efficiency based on the foragers' own activities (here, we use resource depression in a large sense, including behavioral depression and microhabitat depression as well as exploitation depression; see Charnov et al. 1976). Zeder (2012:254) puts it succinctly: "In this way a broad spectrum diet, resource depression, and a decrease in foraging efficiency are all inextricably linked together, imbedded within the foundational assumptions of the OFT world view." Similarly, some have suggested that anthropogenic resource depression is primarily evidenced in zooarchaeological, rather than archaeobotanical, data. The bases of this belief range from differences in human-plant relationships as opposed to human-animal ones to methodological differences (see for instance Campbell and Butler 2010a; Deur and Turner 2005; Peacock and Turner 2000; Smith 2014; Turner et al. 2000). While explanations vary, the impression that archaeobotanists are less likely to identify resource depression than zooarchaeologists is widespread.

However, neither the dominance of resource depression in the zooarchaeological foraging theory literature nor the lack of evidence for resource depression in the archaeobotanical literature have been demonstrated through literature review. In fact, there is some evidence to contradict both these ideas (Codding and Bird 2015). Foraging theory models are used by archaeobotanists and zooarchaeologists to explore themes other than resource depression; indeed, these models have been used archaeologically 
to identify, among other things, instances of prehistoric sustainability (e.g., Campbell and Butler 2010b), cases of environmental rebound (e.g., Jones 2016b), climate-driven changes in resource acquisition (e.g., Broughton et al. 2008), and gendered foraging (e.g., Zeanah 2004). On the other hand, in at least some cases archaeobotanical data have been used to support studies of resource depression (e.g., Simms 1984).

However, this evidence is anecdotal. It does not negate the possibilities that 1) other mechanisms for change identified are adjunct and subordinate to studies of resource depression in the foraging theory literature; and 2) archaeobotanical studies identify resource depression less frequently than zooarchaeological studies.

To explore these possibilities, in this paper we consider the role resource depression and other topics within the zooarchaeology and archaeobotany optimal foraging literature have played over the last 20 years. We assess patterns in publications' analytical foci overall, by subdiscipline, and through time.

\section{Methods}

\section{Data collection}

We searched multiple databases of scholarly publications including Anthropology Plus, Anthrosource, Google Scholar, Web of Knowledge, and WorldCat to identify the relevant literature. Our search criteria required any one of the following phrases: foraging theory, prey choice model, or patch choice model, in addition to some variation of any of the following terms: archaeology, archaeobotany, archaeozoology, paleoethnobotany, or zooarchaeology. Though the exact search differed slightly by database, the search logic can generally be represented as:

("Optimal foraging theory" OR "Prey Choice model" OR "Patch Choice model") AND (archaeology OR archeology OR paleoethno* OR paleobot* OR archaeobot* OR zooarch* OR archaeozoo*)

The search was further limited by year to works published in 1997 or later, and by language to works written in English.

Our search strategy was deliberately broad and emphasized recall over precision (i.e., prioritizing not missing relevant articles over limiting irrelevant results). Therefore, we assessed each article individually to exclude works in which foraging theory was mentioned only in passing, in which zooarchaeological and archaeobotanical data were not the basis of the overall argument, and/or which were purely conceptual. For reasons both practical (we could not obtain all the publications) and abstract (content was not designed as a "publication" per se), we excluded undergraduate theses, conference papers and posters, and non-peer-reviewed reports.

Duplicate results were frequent both within and across databases, with Google Scholar presenting particular challenges. Google Scholar might find the same article on the journal's website, the authors' webpages, social networking sites such as Academia.edu, as well as in one or more institutional repositories. In some cases, the versions retrieved by Google Scholar were uncorrected proofs or otherwise different from the final published form. We used the version of record - that is, the final published formwhenever available.

Despite our focus on recall, our search undoubtedly missed relevant publications. Works not included in the databases searched were, obviously, not found. Additionally, indexing varies across publication and database. If the full text of the publication is not searchable, and the terms we searched for do not appear in the indexed fields (e.g., in title, abstract, keyword, or subject fields), the publication would not be retrieved even if the work was relevant. Finally, in dealing with large results sets with significant duplication, human error was also likely a factor.

Our final dataset contained 244 individual articles, chapters, books, dissertations and theses, representing the work of more than 250 individual authors working on six continents (see supplementary dataset and bibliography). As these data are limited to those authors publishing explicitly within foraging theory, they cannot be used to study trends within zooarchaeology or archaeobotany overall. However, we believe they do provide a reasonable sample to understand the zooarchaeology and archaeobotany foraging theory literature. Citations to these articles are provided in the supplementary files.

\section{Analyses}

We read all publications in the dataset and recorded whether they used zooarchaeological data, archaeobotanical data, or both. "Zooarchaeology" comprised any study using animal (vertebrate or invertebrate) remains as the basis of study, while "archaeobotany" included any study using plant data (pollen, phytoliths, macrobotanical data, etc.). 
Table 1 Descriptions of topical categories used in this analysis.

\begin{tabular}{|c|c|c|}
\hline Category & Examples of topics & Overall $n$ \\
\hline Resource depression & Reduced foraging efficiency due to foragers' own activities & 85 \\
\hline Domestication & $\begin{array}{l}\text { Prey/patch choice approaches to domestication of either (or both) plants } \\
\text { and animals }\end{array}$ & 22 \\
\hline Patch choice/CPF & $\begin{array}{l}\text { Patch choice, central place foraging, and/or marginal value theorem ap- } \\
\text { proaches to understanding subsistence and/or resource use }\end{array}$ & 70 \\
\hline Sustainability & Sustainable use; conservation & 37 \\
\hline $\begin{array}{l}\text { Environment/climate } \\
\text { change }\end{array}$ & $\begin{array}{l}\text { Connection between environmental variables and changes in prey choice } \\
\text { and/or patch use }\end{array}$ & 50 \\
\hline Culture & Costly signaling; gender and risk; niche construction & 39 \\
\hline Method/Theory & Prey ranking systems; patch reconstruction; ethnoarchaeology & 54 \\
\hline
\end{tabular}

We then assigned each publication to at least one broad topical category (Table 1). We developed the categories organically, based on the topics represented in the readings, eventually arriving at seven: anthropogenic resource depression, environmental/ climate change, patch choice/central place foraging, sustainability, domestication, culture and method. We chose not to make these categories mutually exclusive, so as not to underrepresent the presence of resource depression in the literature; a paper could be assigned to more than one category, and indeed most of them were.

The resource depression category included papers which either identified anthropogenic resource depression in a specific instance or which did not rule it out. Conversely, environmental/climate change papers invoked environment as the explanatory mechanism for change. Patch choice and central place foraging were originally separate categories, but so many papers that used one also made use of the other that we decided to collapse them into a single category. The categories of sustainability, domestication, and culture all invoke human activity as a causal mechanism: to be classed as a "sustainability" publication, the paper had to explicitly use that term or claim a demonstration of forager conservation; domestication papers focused on either plant or animal domestication or husbandry, or some combination thereof; and culture papers identified other aspect(s) of human behavior as causal in subsistence patterns, including technology, settlement strategy, costly signaling, and/or gender. Finally, method papers focused on the application of foraging theory to the zooarchaeological or archaeobotanical record.

Jones and Hurley. 2017. Ethnobiology Letters 8(1):35-42
Finally, we considered change in topic representation through time, using four broad time categories: 1997-2004 (number of papers $=49$ ); 2005 -2008 (number of papers $=54$ ); 2009-2012 (number of papers = 76); and 2013-2017 (number of papers = 65). A numerical summary of the categories represented by group can be seen in Table 2; the full analytical dataset and associated bibliography are available as supplementary files.

\section{Results}

There are significantly more zooarchaeological publications $(n=208)$ than archaeobotanical $(n=60)$ in our final dataset. Twenty-four publications (or 10\% of the total dataset) used a combination of zooarchaeological and archaeobotanical data, resulting in some overlap between these two categories. Interestingly, the percentage of papers using a combination of data types remains consistent through time (1997-2004: 10\%; 2005-2008: 8\%; 2009-2012: 11\%; 2013-2017: 11\%).

In the dataset as a whole, anthropogenic resource depression is the most common literature category $(24 \%)$. However, resource depression is by no means as dominant as has sometimes been suggested; many of the other categories are also well-represented. Patch choice/central place foraging analyses are present in $20 \%$ of the publications, and papers in all categories except domestication average over $10 \%$ of the dataset (Figure 1; Table 1).

When zooarchaeological and archaeobotanical publications are considered separately, some interesting patterns emerge. Resource depression is less common in archaeobotanical papers $(19 \%)$ than 
Table 2 Sample size by analytical category.

\begin{tabular}{|c|c|c|c|c|c|c|c|}
\hline Category & Zooarchaeology ${ }^{1}$ & Archaeobotany $^{1}$ & Both $Z A$ and $A B^{1}$ & $\begin{array}{r}1997- \\
2004\end{array}$ & $\begin{array}{r}2005- \\
2008\end{array}$ & $\begin{array}{r}2009- \\
2012\end{array}$ & $\begin{array}{r}2013- \\
2017\end{array}$ \\
\hline $\begin{array}{l}\text { Resource } \\
\text { depression }\end{array}$ & 75 & 20 & 11 & 20 & 20 & 28 & 16 \\
\hline Domestication & 10 & 15 & 4 & 3 & 8 & 3 & 7 \\
\hline Patch choice/CPF & 56 & 18 & 5 & 15 & 13 & 26 & 15 \\
\hline Sustainability & 30 & 10 & 4 & 4 & 11 & 8 & 13 \\
\hline Environment/ & 39 & 14 & 4 & 7 & 16 & 9 & 17 \\
\hline Culture & 33 & 15 & 7 & 11 & 10 & 12 & 8 \\
\hline Method/Theory & 47 & 12 & 5 & 18 & 11 & 11 & 14 \\
\hline
\end{tabular}

${ }^{1}$ The Zooarchaeology and Archaeobotany columns are not mutually exclusive; the number of papers using both methods can be found in the "Both ZA and AB" column.

in zooarchaeological ones (26\%), though not dramatically so (Figure 1). More strikingly, papers focusing on domestication are more common in the archaeobotany literature $(14 \%)$ than in zooarchaeology (3\%) and, conversely, method papers are more common in zooarchaeology $(16 \%)$ than in archaeobotany $(11 \%)$. That the differences in categorical representation between the two subdisciplines are driven by these two categories is confirmed by a Spearman's rank-order correlation: when the categories of domestication and method are excluded, the distributions of categories in the zooarchaeology and archaeobotany literature are significantly correlated $\left(r_{s}=0.90 ; p=0.02\right)$. This suggests that, in this dataset, the differences between the zooarchaeology foraging theory literature and that in archaeobotany are not a result of more zooarchaeologists identifying resource depression; instead, they seem to reflect more foraging-theory oriented archaeobotanists publishing on domestication, while more zooarchaeologists explore methodological issues. The strength of this result suggests as well that the uneven sample sizes between the zooarchaeology and archaeobotany papers are not causing the difference.

The distribution of categories through time highlights another interesting result (Figure 2). While there are variations through time, the most striking trend is the apparent decrease in the number of resource depression papers in the period 2013-2017. Between 1997 and 2012, papers identifying resource depression in some form comprised between 22-29\% of all optimal foraging zooarchaeology and archaeobotany papers. In the period between 2013 and 2017, resource depression papers drop to $18 \%$. A Spearman's rank order correlation suggests a difference in category rank from 1997-2012 and 2013 $-2016\left(\mathrm{r}_{\mathrm{s}}=0.57 ; \mathrm{p}=0.17\right)$.

Another pattern in the chronological analysis concerns the prevalence of method papers. Method papers are relatively frequent in the period 1997-2004 (23\%), diminish in frequency between 2005 and 2012 (2005-2008: 12\%; 2009-2012: 11\%), and then increase between 2013 and 2017 (16\%).

\section{Discussion}

Three major patterns in optimal foraging research in zooarchaeology and archaeobotany emerge from these data: 1) differences between the archaeobotanical and zooarchaeological literature seem to reflect the prevalence of domestication and methods papers, rather than a willingness to identify resource depression; 2) studies identifying prehistoric resource depression appear to be decreasing in frequency in the last few years; and 3) methodoriented papers, which were relatively common between 1997 and 2004, have, after a period of decline, increased again. We discuss each of these findings below.

First, our review suggests identification of resource depression appears in the archaeobotanical foraging theory literature at rates similar to its 


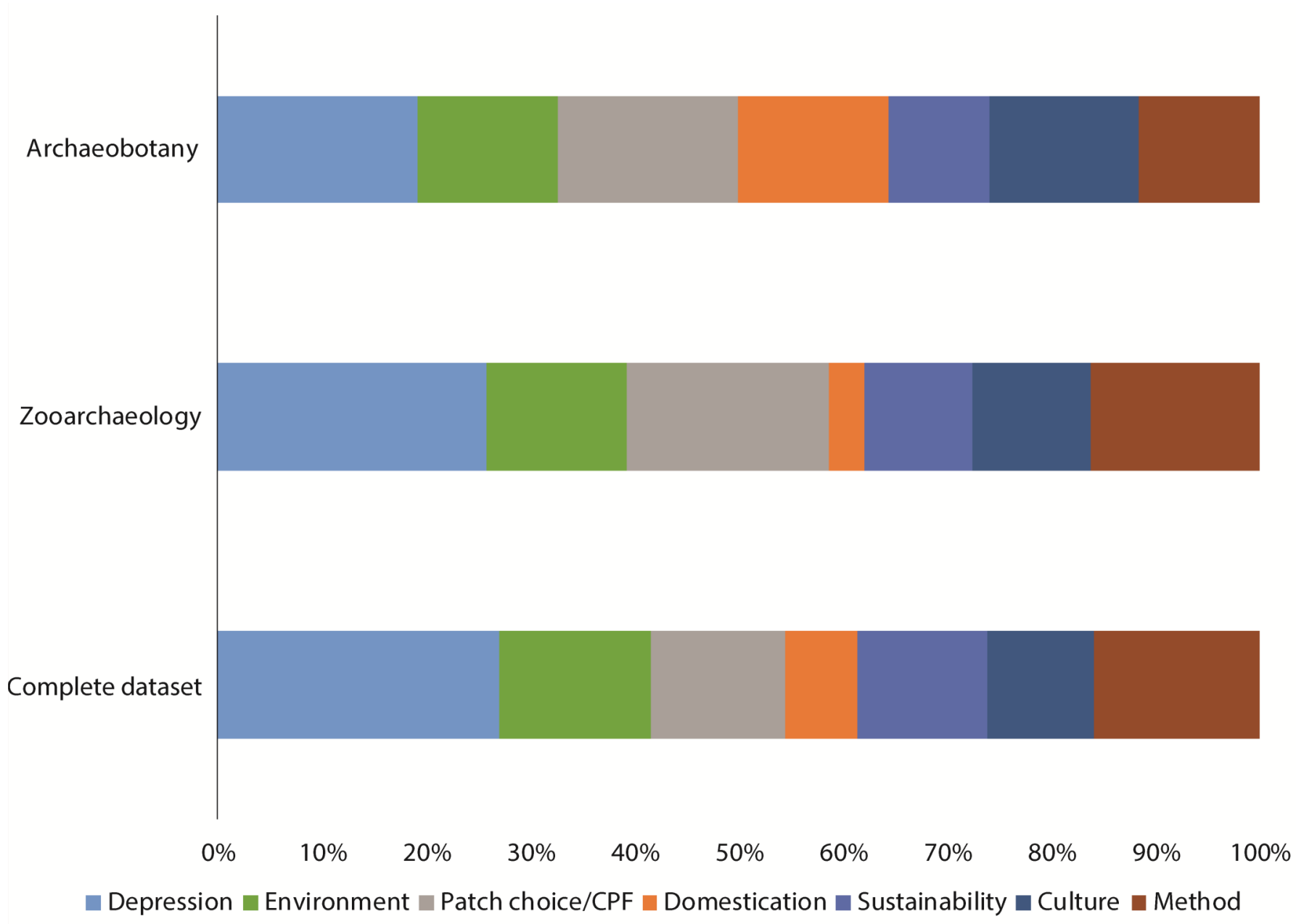

Figure 1 Topics of foraging theory publications in archaeobotany, in zooarchaeology, and in both.

appearance in the zooarchaeological foraging theory literature; the more significant difference in the foraging theory literature between these two disciplines is the prevalence of domestication publications in the archaeobotanical literature and that of methods-oriented papers in the zooarchaeological literature. There are several important caveats to this finding. As discussed in the methods section, our search was limited to foraging theory publications; it did not consider analyses taking place outside of this theoretical framework. The work of archaeobotanists who fully reject optimal foraging theory is thus not represented in this dataset. However, these data do allow us to say resource depression has been identified in both the archaeobotanical and zooarchaeological literature, which has previously been a matter of some debate.
Second, although resource depression has been widely identified in archaeological papers over the last decade, foraging theory has been used to explore many other topics as well. These data show resource depression is far from as dominant in the foraging theory literature as it has been portrayed. In addition, identification of resource depression has decreased in the most recent literature, while papers assigning sustainable practice or environmental change as the causal mechanism for subsistence change have increased. This trend may reflect any of several different factors: it may be a stochastic change, it may be the result of increased funding for climate-related work available from U.S. governmental agencies associated with the American Recovery and Reinvestment Act of 2009, it may be due to foraging theorists responding to critiques. But it is also possible that, in intellectual approaches as in the biological 


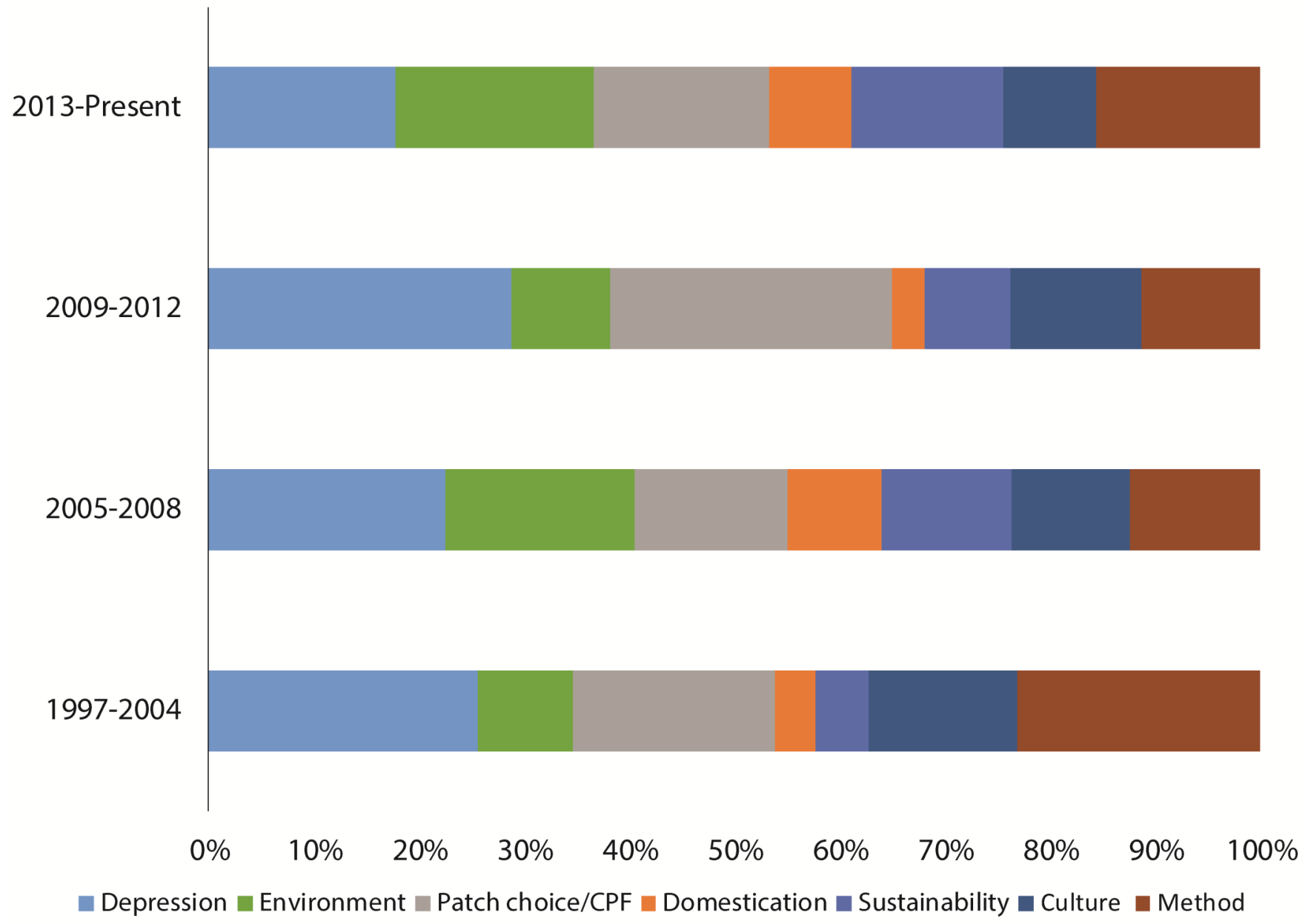

Figure 2 Topics of foraging theory publications from 1997 through 2017.

world, diversity increases with time. Foraging theory in archaeology began with resource depression. Should it be any surprise, thirty years later, that the richness of foraging theory approaches has increased?

The third finding, that method papers are again on the rise in the foraging theory literature, lends some support to the idea that the decrease in identification of resource depression may be at least in part a response to critiques. The prevalence of method papers relatively early in the history of foraging theory in archaeology is reasonable: the first applications of a new approach necessitate methodological conversations and the decline of such publications in later years, as researchers come to agreement on methods, makes sense. However, the recent increase is a surprise. While this may be stochastic variation rather than a true trend, the correlation of these papers with the timing of the publication of critiques associated with niche construction theory (e.g., Gremillion et al. 2014; Mohlenhoff et al. 2015; Smith 2015; Zeder 2015b) is suggestive.

Studies identifying resource depression in the archaeological record have, without a doubt, been one of the major contributions of optimal foraging theory to archaeology more broadly. We do not wish to downplay the importance of such studies. Instead, the goal of this paper has been, quite simply, to address the question of whether studies of resource depression are as dominant in the archaeological optimal foraging literature as critiques often imply. Our data show that in the past two decades, regardless of time of publication or of subdiscipline, resource depression is far from the only application of foraging theory. However, there are numerous questions that remain about how foraging theory is represented in 
zooarchaeology and archaeobotany, which are beyond the scope of this paper but nonetheless intriguing. To what degree is the literature dominated by certain prolific authors? While this dataset shows a wide range of approaches to foraging theory from many different researchers, the influence of specific individuals is apparent in the raw data. Similarly, while the publications assessed here are based on research around the world in a multitude of countries and contexts, there does appear to be bias towards research originating in North America and particularly, Western North America. Is this regional focus changing, and if so, how? Finally, the relationship between foraging theory and niche construction in the literature is not merely one of conflict; indeed, as several of the articles cited earlier in this discussion show, many authors find these to be compatible approaches (e.g., Broughton et al. 2010; Piperno et al. 2017; Stiner and Kuhn 2016). Historical ecology, resilience, and sustainability, similarly, are non-optimality-based approaches used by researchers who also use foraging theory (see discussions in Giovas 2016; Redman 2005; Reitz 2004; Reitz et al. 2009). To what degree are hybrid approaches increasing in the literature?

These questions remain to be answered. For now, however, we can say with certainty that while foraging theory in zooarchaeology and archaeobotany may not be "beyond depression," it is certainly more than depression.

\section{Acknowledgments}

Our thanks to Elic Weitzel and Steve Wolverton for the invitation to participate in the session in which this paper had its origins; to the participants and attendees of the "Foraging Theory: Zooarchaeology and Archaeobotany" session at the 2016 Society for Ethnobiology Meetings in Tucson, Arizona for comments and initial feedback; to the exceptionally helpful comments from four anonymous reviewers; and to the many researchers whose papers we read as part of this project.

\section{Declarations}

Permissions: None declared.

Sources of Funding: None declared.

Conflicts of Interest. None declared.

\section{References Cited}

Broughton, J. M., D. A. Byers, R. A. Bryson, W. Eckerle, and D. B. Madsen. 2008. Did Climatic
Seasonality Control Late Quaternary Artiodactyl Densities in Western North America? Quaternary Science Reviews 27:1916-1937. DOI:10.1016/ j.quascirev.2008.07.005.

Broughton, J. M., M. D. Cannon, and E. J. Bartelink. 2010. Evolutionary Ecology, Resource Depression, and Niche Construction Theory: Applications to Central California Hunter-Gatherers and MimbresMogollon Agriculturalists. Journal of Archaeological Method and Theory 17:371-421. DOI:10.1007/s10816 -010-9095-7.

Campbell, S. K., and V. Butler. 2010a. Fishes and Loaves?: Explaining Sustainable, Long-Term Animal Harvesting on the Northwest Coast Using the "Plant Paradigm". In Archaeology of Anthropogenic Environments, edited by R. M. Dean, pp. 175-203. University of Southern Illinois-Carbondale, Center for Archaeological Investigations Series, Carbondale, IL.

Campbell, S. K., and V. L. Butler. 2010b. Archaeological Evidence for Resilience of Pacific Northwest Salmon Populations and the Socioecological System over the Last 7,500 Years. Ecology and Society 15:17. DOI:10.5751/es-03151150117.

Charnov, E. L., G. H. Orians, and K. Hyatt. 1976. Ecological Implications of Resource Depression. The American Naturalist 110:247-259. DOI:10.1086/283062

Codding, B. F., and D. W. Bird. 2015. Behavioral Ecology and the Future of Archaeological Science. Journal of Archaeological Science 56:9-20. DOI:10.1016/ j.jas.2015.02.027.

Codding, B. F., D. W. Bird, and R. Bliege Bird. 2010. Interpreting Abundance Indices: Some Zooarchaeological Implications of Martu Foraging. Journal of Archaeological Science 37:3200-3210. DOI:10.1016/j.jas.2010.07.020.

Deur, D., and N. J. Turner. 2005. Keeping It Living: Traditions of Plant Use and Cultivation on the Northwest Coast of North America. University of Washington Press, Seattle, WA.

Giovas, C. M. 2016. Though She Be but Little: Resource Resilience, Amerindian Foraging, and Long-Term Adaptive Strategies in the Grenadines, West Indies. The Journal of Island and Coastal Archaeology 11:238-263. DOI:10.1080/15564894.2016.1193572. 
Gremillion, K. J., L. Barton, and D. R. Piperno. 2014. Particularism and the Retreat from Theory in the Archaeology of Agricultural Origins. Proceedings of the National Academy of Sciences 111:6171-6177. DOI:10.1073/pnas.1308938110.

Jones, E. L. 2016a. In Search of the Broad Spectrum Revolution in Paleolithic Southwest Europe. Springer International Publishing, Cham, Switzerland.

Jones, E. L. 2016b. Changing Landscapes of Early Colonial New Mexico: Demography, Rebound, and Zooarchaeology. In Exploring Cause and Explanation: Historical Ecology, Demography, and Movement in the American Southwest, edited by C. L. Herhahn and A. F. Ramenofsky, pp. 73-92. University of Colorado Press, Boulder, CO.

Mohlenhoff, K. A., J. B. Coltrain, and B. F. Codding. 2015. Optimal Foraging Theory and NicheConstruction Theory Do Not Stand in Opposition. Proceedings of the National Academy of Sciences of the United States of America 112:E3093. DOI:10.1073/ pnas.1507637112

Peacock, S. L., and N. J. Turner. 2000. "Just Like a Garden": Traditional Resource Management and Biodiversity Conservation in the Interior Plateau of British Columbia. In Biodiversity and Native America, edited by P. E. Minnis and W. J. Elisens, pp. 133179. University of Oklahoma Press, Norman, OK.

Piperno, D. R., A. J. Ranere, R. Dickau, and F. Aceituno. 2017. Niche Construction and Optimal Foraging Theory in Neotropical Agricultural Origins: A Re-Evaluation in Consideration of the Empirical Evidence. Joumal of Archaeological Science 78:214-220. DOI:10.1016/j.jas.2017.01.001.

Redman, C. L. 2005. Resilience Theory in Archaeology. American Anthropologist 107:70-77. DOI:10.1525/aa.2005.107.1.070.

Reitz, E. J. 2004. "Fishing Down the Food Web": A Case Study from St. Augustine, Florida, USA. American Antiquity 69:63-83. DOI:10.2307/4128348.

Reitz, E. J., I. R. Quitmyer, and R. A. Marrinan. 2009. What Are We Measuring in the Zooarchaeological Record of Prehispanic Fishing Strategies in the Georgia Bight, USA? The Journal of Island and Coastal Archaeology 4:2-36. DOI:10.1080/15564890802349894.

Simms, S. R. 1984. Aboriginal Great Basin Foraging Strategies: An Evolutionary Analysis. Unpublished
Doctoral Dissertation, Department of Anthropology, University of Utah, Salt Lake City, UT.

Smith, B. D. 2014. Failure of Optimal Foraging Theory to Appeal to Researchers Working on the Origins of Agriculture Worldwide. Proceedings of the National Academy of Sciences 111:E2829. DOI:10.1073/pnas.1408208111.

Smith, B. D. 2015. A Comparison of Niche Construction Theory and Diet Breadth Models as Explanatory Frameworks for the Initial Domestication of Plants and Animals. Journal of Archaeological Research 23:215-262. DOI:10.1007/ s10814-015-9081-4.

Speth, J. D. 2013. Thoughts About Hunting: Some Things We Know and Some Things We Don't Know. Quaternary International 297:176-185. DOI:10.1016/j.quaint.2012.12.005.

Stiner, M. C., and S. L. Kuhn. 2016. Are We Missing the "Sweet Spot" between Optimality Theory and Niche Construction Theory in Archaeology? Journal of Anthropological Archaeology 44:177-184. DOI:10.1016/j.jaa.2016.07.006.

Turner, N. J., M. B. Ignace, and R. Ignace. 2000. Traditional Ecological Knowledge and Wisdom of Aboriginal Peoples in British Columbia. Ecological Applications 10:1275-1287. DOI:10.2307/2641283.

Zeanah, D. W. 2004. Sexual Division of Labor and Central Place Foraging: A Model for the Carson Desert of Western Nevada. Journal of Anthropological Archaeology 23:1-32. DOI:10.1016/S0278-4165(03) 00061-8.

Zeder, M. A. 2012. The Broad Spectrum Revolution at 40: Resource Diversity, Intensification, and an Alternative to Optimal Foraging Explanations. Journal of Anthropological Archaeology 31:241-264. DOI:10.1016/j.jaa.2012.03.003.

Zeder, M. A. 2015a. Core Questions in Domestication Research. Proceedings of the National Academy of Sciences 112:3191-3198. DOI:10.1073/pnas.1501711112.

Zeder, M. A. 2015b. Reply to Mohlenhoff Et Al.: Human Behavioral Ecology Needs a Rethink That Niche-Construction Theory Can Provide. Proceedings of the National Academy of Sciences 112:E3094. DOI:10.1073/pnas.1508096112.

Supplementary Files available at ojs.ethnobiology.org/ index.php/ebl/article/view/786 